\title{
Genetic Counseling for Cancer: Technology Promises Better Screening for Hereditary Cancer Patients
}

\author{
Kristin B. Niendorf • Brittany C. Thomas
}

Published online: 19 March 2013

(c) Springer Science+Business Media New York 2013

\begin{abstract}
Approximately 100,000 cases of cancer in the U.S. each year are due to inherited genetic conditions. Scores of hereditary cancer syndromes have been described, associated with nearly every cancer type, and prevention measures have proven to decrease cancer risk. With the advent of new technology, increasingly complex genetic testing options exist. Specially trained genetic counselors coordinate the identification, testing, education and care of patients with hereditary cancer risks. Thus, genetic counselors are at the forefront of the integration of new technology into medical care for hereditary cancer patients. New methods test many more genes, faster and more affordably but with the potential challenges of uninformative or undesired information. Additionally, algorithms using these tests will revolutionize the screening of large numbers of people for hereditary cancer syndromes. Genetic counselors are prepared to offer a broad range of testing schemas as well as provide education regarding the impact of the results for providers, patients and families.
\end{abstract}

Keywords Genetic counseling - Hereditary cancer . Familial cancer - Genetic testing · Universal screening . Lynch syndrome $\cdot$ Risk assessment $\cdot$ Next Generation Sequencing

\footnotetext{
K. B. Niendorf $(\square)$

University of Minnesota, 420 Delaware St. SE, Mayo Mail Code 450, Minneapolis, MN 55455, USA

e-mail: baker603@umn.edu

B. C. Thomas

Department of Medical Genetics, Mayo Clinic College of Medicine, 200 First Street SW, Rochester, MN 55905, USA

e-mail: thomas.brittany@mayo.edu
}

\section{Introduction}

With approximately 1.6 million new cancer diagnoses expected in the United States in 2012, it is estimated that $80,000-160,000$ of these cancers are due to hereditary genetic mutations. The impact of this risk exists not only for the cancer patient but also extends to their families [1]. While the hereditary nature of cancer has long been recognized, identification and testing of at-risk individuals has lagged behind the technology of the testing [2, 3]. Even as early as in 1895 , medical literature had documentation of familial colon cancer, now known to be Lynch syndrome [4]. One hundred years later, the first commercially available hereditary cancer genetic test for mutations in the BRCA1 and BRCA2 genes was developed [5, 6]. Since that time, the field of cancer genetic counseling has expanded in order to identify patients at greatest risk for hereditary cancer and to provide genetic testing and medical management recommendations to reduce the risk of these diseases. Genetic counselors, graduate degree professionals trained in hereditary risk assessment and counseling, have grown in number in order to address the need for practitioners who are knowledgeable about the identification of hereditary cancer syndromes and to assist and educate patients and other health care practitioners alike about the benefits and the potential challenges of genetic testing.

\section{Hereditary Cancer Syndromes Overview}

Nearly all cancer types have known associated hereditary syndromes, and genetic testing exists for many of these causative genes. Over 45 hereditary cancer syndromes have been reported including, but not limited to, those known to increase the risks of breast, ovarian, colon, uterine, 
stomach, endocrine and skin cancers [7•]. One of the most well-described syndromes, hereditary breast-ovarian cancer (HBOC) syndrome, is caused by mutations in the BRCA1 and BRCA2 genes which greatly increase a woman's lifetime risk of breast cancer (43-65\% to age 70) and ovarian cancer (11-39\% to age 70) [8•]. There are also significant risks for subsequent primary cancers after the initial cancer diagnosis, risks to men and, potentially, risks of other cancers such as prostate, melanoma and pancreatic [8•].

Codified medical management recommendations exist for individuals who test positive for a mutation in these genes, including intensive and earlier cancer screening as well as prophylactic surgery and other preventative measures. The National Comprehensive Cancer Network (NCCN) recommends that known BRCA mutation carriers have annual mammography and breast MRI starting at age 25 and consideration of risk-reducing salpingo-oophorectomy between 35 and 40 years of age, after completion of childbearing [9]. Other screening, surgical and chemopreventive measures are proposed as well by the NCCN and other agencies. Studies prove that these measures can dramatically reduce the risk of cancer in these high-risk families. For example, prophylactic salpingo-oophorectomy reduces the risk of ovarian cancer by $80 \%$ and the risk of breast cancer by approximately $50 \%$ in BRCA $1 / 2$ carriers [10].

Another well-studied hereditary cancer syndrome, Lynch syndrome, is caused by mutations in the mismatch repair (MMR) genetic pathway, typically mutations in the MLH1 and MSH2 genes and less frequently in the MSH6 and PMS2 genes [11]. Lynch syndrome confers a lifetime risk of colorectal cancer of 30-74\% and up to $60 \%$ risk of endometrial cancer in women [12••]. Other extra-colonic cancer risks exist as well. The NCCN also provides management recommendations for individuals with Lynch syndrome including colonoscopy beginning at ages 20-25 (or 2-5 years before the earliest cancer diagnosis in the family) to be performed every 1-2 years [13]. Cost effectiveness analyses support population screening for Lynch syndrome given the benefits of screening and other riskreducing measures $[15,16]$.

Given the clear benefits of identifying and managing patients with hereditary cancer syndromes, several groups have proposed recommendations for screening populations for these diseases. The American Society of Clinical Oncology (ASCO) in 1996 outlined recommendations regarding the offering of genetic testing for BRCA1 and BRCA2, and in recent updates these recommendations included pre- and post-test genetic counseling [17-19]. In 2009, the initiative, Evaluation of Genomic Applications in Practice and Prevention (EGAPP), recommended that all newly diagnosed colorectal cancer patients be evaluated for Lynch syndrome [14]. This has resulted in the adoption of universal screening of colorectal tumor samples across many institutions. Several consortia have also recommended screening for individuals with cancer via family history characteristics or medical/pathological features or both including the annually updated National Comprehensive Cancer Network (NCCN) Clinical Guidelines (http://www. nccn.org/professionals/physician_gls/f_guidelines.asp).

While these screening regimens will identify more at-risk individuals and families, recently developed technology, such as targeted gene panels, will increase the specificity for detecting those mutations conferring risk in these individuals. Genetic counselors are well prepared to understand the promise of these technologies as well as the potential limitations as they are at the cutting edge of translation of genetic discoveries to patients.

\section{The Use of Targeted Gene Panels for Hereditary Cancer Syndrome Evaluation}

Genetic testing laboratories generally offer a varied menu of comprehensive testing options for the evaluation of patients suspected of having a hereditary predisposition to cancer. The standard technology used to assess for disease-causing mutations within single genes associated with hereditary cancer risk is direct Sanger sequencing. In addition, many laboratories offer methods designed to detect large deletions and duplications of genetic material within a gene which may also be disease-causing. Together, Sanger sequencing and large deletion/duplication assays offer the most sensitive methods for detecting mutations in a single gene, to date. However, this testing is limited in that only one gene is screened per test. Thus, if more than one gene potentially explains a patient's history of cancer(s), multiple tests may be required before the causative mutation is detected. This single testing strategy increases the time and cost associated with the genetic testing.

Genetic testing is often used in a clinical setting as a complement to a detailed and thorough family history and both are crucial for the identification and diagnosis of hereditary cancer syndromes in families. Recognition of specific combinations of cancer types as well as other informative non-cancer related features facilitates the identification of the most likely hereditary cancer syndrome in a given family. This directs the selection of genes to consider for genetic testing. For instance, a family history that includes several individuals with diffuse gastric cancer and lobulartype breast cancer is highly indicative of Hereditary Diffuse Gastric Cancer associated with mutations in the $\mathrm{CDH} 1$ gene [20]. If the patient or family exhibits features which are highly suggestive of a specific hereditary cancer syndrome, the decision to pursue genetic testing may be straightforward. However, because many cancer types, such as colon 
cancer, are included in the tumor spectrum of several hereditary cancer syndromes, it is common to consider multiple genes for genetic testing, increasing the complexity of the genetic risk assessment, the genetic counseling process and patient decision-making.

While some providers and patients chose to test more than one gene at a time, gene testing in a sequential manner is the most cost-effective strategy available, allowing for clinicians to choose and test the most likely gene(s) involved. If the initial test(s) is negative, the clinician and patient may elect to proceed to the next most likely gene on the list of differential diagnoses. Once a mutation is identified, family members have the option of undergoing predictive testing to clarify their own risk for cancer, leading to early detection and prevention of cancer in unaffected individuals. By taking a step-wise approach, the patient avoids incurring unnecessary cost if a mutation is identified early in at-risk family members.

There are several disadvantages to sequential testing, however. Patients can often become discouraged or experience test fatigue if serial genetic testing does not provide a positive result and may decide to discontinue the genetic testing process before all options have been exhausted. Cost can become prohibitive as well, specifically if insurance coverage for genetic testing is limited or not available. The out-of-pocket cost to evaluate a single gene is currently on average $\$ 1,000-\$ 2,000$. Therefore, evaluation for multiple genes in a sequential manner quickly becomes a significant financial burden to the patient. Knowing at what point to cease testing also can be problematic and involves striking a balance between the patient's motivation for testing and the likelihood of yielding an informative result. Therefore, the genetic counseling process with accurate clinical evaluation and risk assessment are critical for facilitating the most appropriate and feasible genetic testing for each patient when multiple genes are being considered.

Recently, Next Generation Sequencing (NGS) technologies have allowed genetic testing laboratories the ability to offer multi-gene panels at reasonable cost to patients, thereby introducing a completely alternative approach to current genetic testing strategies. The NGS is a highthroughput technology that enables massive parallel sequencing of multiple genes simultaneously. Coupled with methods for detecting large deletions and duplications, these technologies provide a highly sensitive option to concurrently hereditary cancer [21•].

The NGS-based gene panels are very appealing as they offer a comprehensive testing option at relatively low cost, while eliminating the complexities and fatigue associated with serial or algorithmic testing. A gene panel is especially attractive for cases in which a specific hereditary cancer syndrome is not apparent in a family, but hereditary risk is still suspected. The ability to test multiple genes simultaneously also enables clinicians to account for phenotypic overlap which may not yet be appreciated or described within the literature. For instance, new data suggests that breast and other cancers not traditionally part of the Lynch syndrome tumor spectrum may now be associated with increased risk in Lynch syndrome patients [22•]. Therefore, targeted-gene panels optimize the chance of finding a gene mutation in a family by providing the opportunity to test several genes associated with a specific indication, for which the phenotypic overlap and risks are yet to be fully developed or described.

While the advantages of targeted-gene panels are immediately apparent, there are several limitations to this testing approach which present new challenges to genetic counseling for hereditary cancer syndromes. Pre-test genetic counseling is significantly impacted by the volume of testing that is performed simultaneously. Discussing each of the individual syndromes on a large gene panel and the implications of a positive and negative test result for each is timeconsuming and potentially confusing for the patient. As informed consent is a key component of the genetic testing process, this posits the question of: "What suffices as informed consent for this type of test?". Clinical guidelines regarding the ascertainment of informed consent were designed for single-gene tiered approaches; however, there are currently no guidelines available specific to large gene panels [17-19].

Of additional concern is the higher likelihood for uninformative or undesired results, such as variants of uncertain clinical significance and alterations detected in low-risk or low-penetrant genes which have questionable or unknown clinical risk. Currently, patients and clinicians are at the mercy of testing laboratories with regards to which genes are chosen for inclusion on a specific panel, some of which may be of limited or unproven clinical utility, as there are currently no laboratory guidelines regarding targeted-gene panels. This presents a difficult genetic counseling situation if the clinical applicability of certain results is questionable as it has the potential to oppose current recommendations on genetic testing for hereditary cancer [7•, 17-19].

Finally, large gene panels do not replace all genetic testing for hereditary cancer syndromes. Current hereditary cancer panels are not able to assess for tumor characteristics such as microsatellite instability, a hallmark feature of Lynch syndrome. This test is useful in determining if a mismatch repair (MMR) defect is present within a tumor, thereby differentiating between true-negative and false-negative results by molecular methods. Similarly, with the discovery of epimutations such as promoter hypermethylation of the KLLN gene recently associated with Cowden syndrome, not all germline causes of hereditary cancer will be detectable by the NGS methods [23]. 
Table 1 Advantages and Disadvantages of step-wise versus multi-gene panel testing

\begin{tabular}{|c|c|}
\hline Advantages & Disadvantages \\
\hline \multicolumn{2}{|l|}{ Step-wise testing } \\
\hline $\begin{array}{l}\text { More cost-effective if considering limited } \\
\text { number of genes }\end{array}$ & $\begin{array}{l}\text { Relies on accurate reporting of diagnosis and clinical features in family members to target } \\
\text { correct gene(s) for testing }\end{array}$ \\
\hline Minimizes unnecessary testing & Increased financial burden if multiple genes are being considered \\
\hline Lower risk for uncertain significance result(s) & Test fatigue if serial testing is performed \\
\hline \multicolumn{2}{|l|}{ Next generation sequence testing } \\
\hline $\begin{array}{l}\text { More comprehensive; optimizes chance of } \\
\text { detecting a germline mutation }\end{array}$ & Informed consent and counseling more time-consuming \\
\hline $\begin{array}{l}\text { More cost-effective if considering multiple } \\
\text { genes }\end{array}$ & Higher risk for variants of uncertain significance and/or alterations in genes of uncertain risk \\
\hline $\begin{array}{l}\text { Allows for phenotypic overlap between } \\
\text { syndromes }\end{array}$ & $\begin{array}{l}\text { Unable to incorporate other recommended methods of evaluation, such as tumor-based } \\
\text { analysis (e.g., MSI/IHC), detection of epimutations (e.g., promoter methylation), and PCR } \\
\text { methods to bypass pseudogenes }\end{array}$ \\
\hline
\end{tabular}

While NGS and targeted-gene panels have great potential to improve evaluation and diagnosis of hereditary cancer syndromes, new ways of counseling and education will be necessary to incorporate these technologies into current clinical practice. The advantages and disadvantages of step-wise testing versus NGS panels are described in the Table 1 .

While targeted gene panels will increase the detection rate of mutations in hereditary cancer syndrome carriers, there is also a need to more throughly identify patients in need of this testing. The universal screening for Lynch syndrome recommended in the EGAPP statement is one of the first examples of wide-scale screening for a hereditary cancer syndrome [11, 14].

\section{Universal Screening for Lynch Syndrome}

Lynch syndrome is characterized primarily by significantly increased risks for colorectal and endometrial cancer but there are elevated risks for other cancers such as gastric, ovarian, hepatobiliary, and sebaceous adenocarcinomas [22•, 24, 25]. While Lynch syndrome is molecularly defined by the presence of a mutation within one of four mismatch repair (MMR) genes (MLH1, MSH2, MSH6 and PMS2), recently, large deletions involving the EPCAM gene have also been identified as a cause of Lynch syndrome [26]. Genetic evaluation for Lynch syndrome may involve testing all four MMR genes until a germline mutation is identified. However, the discovery of a distinct tumor phenotype called microsatellite instability (MSI), detected in DNA extracted from colon tumors, resulted in an alternative testing strategy for Lynch syndrome [27, 28]. The MSI is defined by a discrepancy in the number of mono- and di-nucleotide repeat sequences observed between isolated normal and tumor DNA. The recognition that defects within the MMR pathway lead to the resultant MSI phenotype in nearly all tumors from individuals with Lynch syndrome led to an alternative first-tier option for the evaluation of patients suspected of having Lynch syndrome. Since the discovery of MSI, a complementary test called immunohistochemistry (IHC) was developed to assess for the presence or absence of MLH1, MSH2, MSH6 and PMS2 protein expression within colorectal tumors. Together, MSI and IHC performed on colorectal and endometrial tumors provide a highly sensitive assay for the detection of Lynch syndrome patients [29]. Current national guidelines proposed by both the NCCN and the National Society of Genetic Counselors (NSGC) with the Collaborative Group of the Americas on Inherited Colorectal Cancer (CGA-ICC) recommend MSI and IHC analyses if a tumor is available as the initial screen for Lynch syndrome in a clinic-based high-risk population

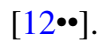

Several proposed sets of criteria and guidelines, such as the Amsterdam Criteria I and II and the Bethesda and revised Bethesda guidelines, have been used in the clinical setting for the ascertainment of families with the highest likelihood of having Lynch syndrome [30-33]. Both Amsterdam Criteria I and II stratify families based on age of onset and incidence of cancer among closely related individuals in a family. The revised Bethesda guidelines were subsequently developed to incorporate specific histopathologic characteristics which tend to be observed with greater frequency in tumors with high levels of MSI than microsatellite stable (MSS) tumors.

However, despite the iterations of proposed clinical criteria and guidelines, a significant proportion of Lynch syndrome patients will fail to be recognized and referred for genetic counseling and risk assessment as awareness of these clinical criteria among non-genetics providers is low [34-36]. Additionally, the Amsterdam Criteria and 
Bethesda Guidelines, while highly sensitive in the clinically ascertained group, fail to identify Lynch syndrome patients whose personal and family histories fall outside the proposed criteria. The sensitivity of the original Amsterdam Criteria I ranges from 54 to $91 \%$ and 38 to $87 \%$ for the less stringent Amsterdam Criteria II, depending on the MMR gene involved [24, 37, 38]. The Bethesda and revised Bethesda Guidelines also fail to detect a portion of Lynch syndrome cases among a population selected for high risk colon cancer [24, 38, 39]. Therefore, screening based on the family history of cancers alone will miss a significant portion of patients with Lynch syndrome.

Due to the suboptimal referral patterns of clinicians and incomplete sensitivity of proposed clinical criteria for the purposes of identifying candidates for genetic counseling, an initiative to screen all cases of colon cancer in the general population regardless of age has been adopted by institutions across the United States within the last several years and was endorsed by the Evaluation of Genomic Applications in Practice and Prevention (EGAPP) in 2009 based on the probable reduction in morbidity and mortality to family members of Lynch syndrome patients [14]. Given that increased surveillance leads to a decrease in colon cancer and subsequent death, identification of at risk individuals in whom targeted surveillance strategies can be initiated is a desired outcome. The clinical sensitivity of MSI versus IHC is nearly equivalent and, therefore, either method is suitable; however, IHC not only demonstrates the presence or absence of defective mismatch repair, it also directs downstream germline testing by indicating which of the four MMR genes is most likely to be deficient [29]. Therefore, IHC followed by a referral for genetic counseling may be a preferred strategy.

About $15-20 \%$ of all colorectal cancer in an unselected population will be MSI-H and demonstrate absence of protein expression by IHC. A majority of these MMR deficient tumors are due to sporadic inactivation of the MLH1 gene by promoter hypermethylation [40]. Studies have demonstrated a strong correlation between increasing age and the presence of MLH1 promoter hypermethylation, thus explaining the high incidence of this finding in an unselected population [41]. As such, the EGAPP working group also recommended evaluation for the BRAF V600E mutation which is associated with a nearly $100 \%$ specificity for sporadic colon cancers due to MLH1 promoter hypermethylation [42]. Therefore, testing for the presence of this specific BRAF mutation provides a feasible option for ruling out sporadic cases of colon cancer ascertained by universal screening.

The adoption of universal screening of colorectal tumors for MSI and/or IHC, has also led to the advent of screening all or a subset of endometrial tumors at some institutions. The EGAPP working group could not substantiate similar benefit in endometrial tumors and, therefore, did not recommend universal screening in this clinical population. The false-negative rate among clinically-ascertained highrisk patients and an unselected general population of colon cancers would not be expected to differ greatly [22•]. However, the false-positive rates are higher in all-comers as opposed to patients pre-selected based on personal and family history, as demonstrated by the higher proportion of individuals with absent MLH1 protein expression, presumably due to somatic MLH1 promoter hypermethylation, in the unselected group [22•]. Additionally, the falsepositive rates associated with the other two proteins, $\mathrm{MSH} 2$ and MSH6, will likely increase as well. This is supported by reports in the dermatopathology literature which have recently suggested a sporadic etiology for the absence of MSH2 and MSH6 in sebaceous neoplasms [43]. The larger the number of older-onset and isolated cases of colon and endometrial tumors evaluated by programs like universal Lynch syndrome screening, the higher the likelihood of identifying other sporadic tumor etiologies. Until these sporadic causes are better delineated, unexplained falsepositive results may be prevalent among unselected screening populations. False-positives can lead to feelings of anxiety in patients as well as over-screening in family members. Appropriate genetic counseling false positive results in screening populations is needed.

General population screening for hereditary disease is rare apart from newborn screening for metabolic disorders. Therefore, considerations such as economic burden, social benefit, and informed consent as discussed by the EGAPP working group, will continue to be sources of debate among institutions considering the adoption of universal screening for Lynch syndrome.

\section{Conclusion}

Advances in technology hold the promise of identifying more patients and families at hereditary cancer risk. Targeted gene panels using next generation sequencing utilizes cutting-edge technology to evaluate multiple genes at a time for the purposes of identifying causative mutations associated with known hereditary cancer syndromes. Similarly, universal screening for Lynch syndrome has expanded the world of tumor-based testing in an effort to capture as many individuals with Lynch syndrome as possible. These methods are sure to accelerate the identification of patients in need of genetic risk assessment and counseling as well as provide more comprehensive genetic testing results.

All of these advances in cancer genetics will increase the sensitivity of identifying patients at increased risk for cancer due to heritable susceptibility and subsequently increase the demand for cancer genetics services. Cancer 
genetic counselors will be pivotal in ensuring appropriate utilization of these new advances and technologies as well as the education of patients and providers about their benefits and limitations. While these advances intimately affect the provision of cancer genetic counseling, they also promise to alter the provision of cancer care throughout the medical system. Since cancer is one of the most common causes of death, optimizing the chances of detecting the portion of cases due to hereditary syndromes has the potential to significantly reduce the overall risk of cancer morbidity and mortality.

Disclosure KB Niendorf declares no conflicts of interest and $\mathrm{BC}$ Thomas declares no conflicts of interest.

\section{References}

Papers of particular interest, published recently, have been highlighted as:

- Of importance

-• Of major importance

1. Siegel R, Naishadham D, Jemal A. Cancer statistics, 2012. CA Cancer J Clin. 2012;62:10-29.

2. Meyer LA, Anderson ME, Lacour RA, et al. Evaluating women with ovarian cancer for BRCA1 and BRCA2 mutations: missed opportunities. Obstet Gynecol. 2010;115:945-52.

3. Bellcross CA, Kolor K, Goddard KA, et al. Awareness and utilization of BRCA1/2 testing among U.S. primary care physicians. Am J Prev Med. 2011;40:61-6.

4. Douglas JA, Gruber SB, Meister KA, et al. History of molecular genetics of Lynch syndrome in family G: a century later. JAMA. 2005;294:2195-202.

5. Friedman LS, Ostermeyer EA, Szabo CI, et al. Confirmation of BRCA1 by analysis of germline mutations linked to breast and ovarian cancer in ten families. Nat Genet. 1994;8:399-404.

6. Collins N, McManus R, Wooster R, et al. Consistent loss of the wild type allele in breast cancers from a family linked to the BRCA2 gene on chromosome 13q12-13. Oncogene. 1995;10: 1673-5.

7. - Riley BD, Culver JO, Skrzynia C, et al. Essential elements of genetic cancer risk assessment, counseling and testing: updated recommendations of the National Society of Genetic Counselors. J Genet Counsel. 2012;21:151-161. This paper, from the National Society of Genetic Counselors [NSGC (United States)], provides current Practice Guidelines on Genetic Counseling for Cancer.

8. - Berliner JL, Fay AM, Cummings SA, Burnett B, Tillmanns T. NSGC practice guideline: risk assessment and genetic counseling for hereditary breast and ovarian cancer. J Genet Couns. 2013;22:155-63. This paper, from the National Society of Genetic Counselors [NSGC (United States)], provides current Practice Guidelines on Genetic Counseling for Hereditary Breast and Ovarian Cancer.

9. National Comprehensive Cancer Network guidelines for genetic/ familial high-risk assessment: breast and ovarian. http://www. nccn.org/patients/patient_gls/_english/pdf/NCCN\%20Breast\%20 Guidelines.pdf. Accessed 23 Feb 2013.

10. Rebbeck TR, Kauf ND, Domcheck S. Meta-analysis of Risk Reduction Estimates Associated With Risk-Reducing Salpingo- oophorectomy in BRCA1 or BRCA2 Mutation Carriers. J Natl Cancer Inst. 2009;101:80-7.

11. Palomaki GE, McClain MR, Melillo S, et al. EGAPP Supplementary evidence review: DNA Testing strategies aimed at reducing morbidity and mortality from Lynch syndrome. Gen Med. 2009;11:42-65.

12. • Weissman SM, Burt R, Church J, et al. Identification of individuals at risk for Lynch syndrome using targeted evaluations and genetic testing: National Society of Genetic Counselors and the Collaborative Group of the Americas on Inherited colorectal cancer joint practice guideline. J Genet Counsel. 2012; 21:484-493. This joint recommendation from the National Society of Genetic Counselors [NSGC (United States)] and the Collaborative Group of the Americas on Inherited Colorectal Cancer (GCA-ICC) outlines protocols for identifying and testing patients for Lynch syndrome.

13. National Comprehensive Cancer Network guidelines for colorectal cancer screening version 2:2012. http://www.nccn.org/ professionals/physician_gls/pdf/colorectal_screening.pdf. Accessed 23 Feb 2013.

14. Recommendations from the EGAPP Working Group. genetic testing strategies in newly diagnosed individuals with colorectal cancer aimed at reducing morbidity and mortality from Lynch syndrome in relatives. Genet Med. 2009;11:35-41.

15. Ladabaum U, Wang G, Terdiman J, et al. Strategies to identify the Lynch syndrome among patients width colorectal cancer: a cost-effectiveness analysis. Ann Intern Med. 2011;155:69-79.

16. Mvundura M, Grosse SD, Hampel H, et al. The cost effectiveness of genetic testing strategies for Lynch syndrome among newly diagnosed patients with colorectal cancer. Genet Med. 2010;12:93-104.

17. Statement of the American Society of Clinical Oncology. genetic testing for cancer susceptibility, Adopted on February 201996. J Clin Oncol. 1996;14:1730-17366. discussion 7-40.

18. American Society of Clinical Oncology policy statement update. genetic testing for cancer susceptibility. J Clin Oncol. 2003; 21:2397-406.

19. Robson ME, Storm CD, Weitzel J, et al. American Society of Clinical Oncology policy statement update: genetic and genomic testing for cancer susceptibility. J Clin Oncol. 2010;28:893-901.

20. Fitzgerald RC, Hardwick R, Huntsman D, et al. Hereditary diffuse gastric cancer: updated consensus guidelines for clinical management and directions for future research. J Med Genet. 2010;47:436-44.

21. - Pritchard CC, Smith C, Salipante SJ, et al. ColoSeq provides comprehensive lynch and polyposis syndrome mutational analysis using massively parallel sequencing. J Mol Diagn. 2012;14:357-366. This paper describes the first targeted hereditary colon cancer panel (ColoSeq) by Next Generation Sequencing technologies.

22. - Engel C, Loeffler M, Steinke V, et al. Risks of less common cancers in proven mutation carriers with lynch syndrome. J Clin Oncol. 2012;30:4409-4415. This paper further defines the risk for Lynch syndrome-related cancers through evaluation of a very large cohort of known Lynch syndrome mutation carriers; risks for less common cancers, such as breast cancer, were described.

23. Ngeow J, Mester J, Rybicki LA, et al. Incidence and clinical characteristics of thyroid cancer in prospective series of individuals with Cowden and Cowden-like syndrome characterized by germline PTEN, SDH, or KLLN alterations. J Clin Endocrinol Metab. 2011;96:E2063-71.

24. Vasen HF, Moslein G, Alonso A, et al. Guidelines for the clinical management of Lynch syndrome (hereditary non-polyposis cancer). J Med Genet. 2007;44:353-62.

25. Lindor NM, Rabe K, Petersen GM, et al. Lower cancer incidence in Amsterdam-I criteria families without mismatch repair 
deficiency: familial colorectal cancer type X. JAMA. 2005; 293:1979-85.

26. Ligtenberg MJ, Kuiper RP, Chan TL, et al. Heritable somatic methylation and inactivation of MSH2 in families with Lynch syndrome due to deletion of the $3^{\prime}$ exons of TACSTD1. Nat Genet. 2009;41:112-7.

27. Aaltonen LA, Peltomaki P, Leach FS, et al. Clues to the pathogenesis of familial colorectal cancer. Science. 1993;260:812-6.

28. Ionov Y, Peinado MA, Malkhosyan S, Shibata D, Perucho M. Ubiquitous somatic mutations in simple repeated sequences reveal a new mechanism for colonic carcinogenesis. Nature. 1993;363:558-61.

29. Baudhuin LM, Burgart LJ, Leontovich O, et al. Use of microsatellite instability and immunohistochemistry testing for the identification of individuals at risk for Lynch syndrome. Fam Cancer. 2005;4:255-65.

30. Umar A, Boland CR, Terdiman JP, et al. Revised Bethesda Guidelines for hereditary nonpolyposis colorectal cancer (Lynch syndrome) and microsatellite instability. J Natl Cancer Inst. 2004;96:261-8.

31. Vasen HF, Mecklin JP, Khan PM, Lynch HT. The international collaborative group on hereditary non-polyposis colorectal cancer (ICG-HNPCC). Dis Colon Rectum. 1991;34:424-5.

32. Rodriguez-Bigas MA, Boland CR, Hamilton SR, et al. A National Cancer Institute workshop on hereditary nonpolyposis colorectal cancer syndrome: meeting highlights and Bethesda guidelines. J Natl Cancer Inst. 1997;89:1758-62.

33. Vasen HF, Watson P, Mecklin JP, Lynch HT. New clinical criteria for hereditary nonpolyposis colorectal cancer (HNPCC, Lynch syndrome) proposed by the International Collaborative group on HNPCC. Gastroenterology. 1999;116:1453-628.

34. Trano G, Wasmuth HH, Sjursen W, Hofsli E, Vatten LJ. Awareness of heredity in colorectal cancer patients is insufficient among clinicians: a Norwegian population-based study. Colorectal Dis. 2009;11:456-61.

35. Lindor NM, McMaster ML, Lindor CJ, Greene MH. Concise handbook of familial cancer susceptibility syndromes-second edition. J Natl Cancer Inst Monogr. 2008;38:1-93.

36. Tan YY, McGaughran J, Ferguson K, et al. Improving identification of lynch syndrome patients: A comparison of research data with clinical records. Int J Cancer. 2013. doi:10.1002/ijc.27978.

37. Kievit W, de Bruin JH, Adang EM, et al. Current clinical selection strategies for identification of hereditary non-polyposis colorectal cancer families are inadequate: a meta-analysis. Clin Genet. 2004;65:308-16.

38. Sjursen W, Haukanes BI, Grindedal EM, et al. Current clinical criteria for Lynch syndrome are not sensitive enough to identify MSH6 mutation carriers. J Med Genet. 2010;47:579-85.

39. Hampel H, Frankel WL, Martin E, et al. Feasibility of screening for Lynch syndrome among patients with colorectal cancer. J Clin Oncol. 2008;26:5783-8.

40. Cunningham JM, Christensen ER, Tester DJ, et al. Hypermethylation of the hMLH1 promoter in colon cancer with microsatellite instability. Cancer Res. 1998;58:3455-60.

41. Kakar S, Burgart LJ, Thibodeau SN, et al. Frequency of loss of hMLH1 expression in colorectal carcinoma increases with advancing age. Cancer. 2003;97:1421-7.

42. Domingo E, Laiho $\mathrm{P}$, Ollikainen $\mathrm{M}$, et al. BRAF screening as a low-cost effective strategy for simplifying HNPCC genetic testing. J Med Genet. 2004;41:664-8.

43. Roberts ME, Riegert-Johnson DL, Thomas BC, et al. Screening for Muir-Torre syndrome using mismatch repair protein immunohistochemistry of sebaceous neoplasms. J Genet Couns. 2012. doi:10.1007/s10897-012-9552-4. 